\title{
Curaderm, the Long-Awaited Breakthrough for Basal Cell Carcinoma
}

\author{
T. Chase, K. E. Cham, B. E. Cham \\ Australasian Medical Research, Port Vila, Republic of Vanuatu \\ Email: bill.cham@gmail.com
}

How to cite this paper: Chase, T., Cham, K.E. and Cham, B.E. (2020) Curaderm, the Long-Awaited Breakthrough for Basal Cell Carcinoma. International Journal of Clinical Medicine, 11, 579-604. https://doi.org/10.4236/ijcm.2020.1110050

Received: September 18, 2020

Accepted: October 25, 2020

Published: October 28, 2020

Copyright $\odot 2020$ by author(s) and Scientific Research Publishing Inc. This work is licensed under the Creative Commons Attribution International License (CC BY 4.0).

http://creativecommons.org/licenses/by/4.0/

(c) (i) Open Access

\begin{abstract}
Background: Basal cells form a continuous cell layer at the bottom of the epidermis, which is the outermost layer of the skin. Basal cell carcinoma occurs when a mutation occurs in the DNA of a basal cell. The mutation inhibits apoptosis-the programmed cell death mechanism. The cell continues to multiply but does not die, resulting in a change in the skin, such as a growth or sore that will not heal. Basal cell carcinoma is the most common form of skin cancer and the most frequently occurring form of all cancers. Key words searched for the database of this communication were: Curaderm, BEC 5, cancer, skin cancer, basal cell carcinoma, nonmelanoma skin cancer, solamargine, solasonine and solasodine glycosides. Treatments: Several types of treatments are available to remove or destroy basal cell carcinoma. All currently used treatments are indiscriminate and also remove or destroy normal skin cells resulting in compromised cosmetic outcomes. Development of Curaderm Pharmacotherapy: Curaderm pharmacotherapy discriminates and specifically activates apoptosis at the molecular level in cancer cells but not in normal cells. Accordingly, Curaderm pharmacotherapy for basal cell carcinoma effectively and safely treats virtually all types, sizes and lesion locations. This review describes studies from the inception of Curaderm pharmacotherapy and covers the discovery of the anti-cancer effects, mode of action, preclinical, clinical and field applications with emphasis on efficacy, safety, compliance, tolerance, cost effectiveness and especially cosmetic outcome. In 2018 Curaderm was approved by the European Health Authorities as a Medical Device Class 1 for the indication "Topical Treatment with Keratolytic Action, and Antineoplastic Activity in the Treatment and Healing of Localized Basal Cell Carcinoma of the Skin".
\end{abstract}

\section{Keywords}

Basal Cell Carcinoma, Skin Cancer, BEC, Solamargine, Curaderm, Topical, Pharmacotherapy 


\section{Introduction}

Basal cell carcinoma (BCC) is the most common form of skin cancer and the most frequently occurring form of all cancers. In the U.S. alone, more than 4 million cases are diagnosed each year. BCC is a disease of global health importance that causes substantial psychosocial impacts and requires considerable investment in terms of treatment and technologies. Conventional therapies have several drawbacks, thus, a long-awaited treatment breakthrough for BCC is very welcome.

\subsection{Aetiology}

BCC begins in the basal cells-a cell layer at the bottom of the epidermis which is the outermost layer of the skin that produces new skin cells as old ones die off. BCC occurs when a mutation occurs in the DNA of one or more basal cells. The process of creating new skin cells is controlled by the basal cell's DNA. The DNA contains the instructions that tell the cell how to behave. A mutation can tell the cell to multiply more rapidly and continue to grow when it would normally die. For every normal cell, there is a time to live and a time to die. Roughly 50 billion cells are born and die each day in humans.

Under normal conditions, a programmed sequence of events, known as apoptosis, leads to the elimination of cells without releasing harmful substances into the surrounding area. Basal cells in the basal cell layer (stratum basale) of the skin are also referred to as basal keratinocytes. Keratinocyte apoptosis plays a critical role in regulating epidermal development and restraining carcinogenesis.

Apoptosis balances proliferation to maintain epidermal thickness and contributes to stratum corneum formation. When basal cells are mutated, apoptosis may not work correctly. Cells that should be eliminated may persist and become immortal, such as the case with BCC.

BCC starts when basal cells grow out of control and crowd out normal cells. In BCC, the process of apoptosis is defunct but cell division is intact resulting in excessive numbers of cancer cells, resulting in a change in the skin, such as a growth or a sore that will not heal.

With BCC, these lesion changes in the skin may express one of the following characteristics:

- A pearly white, skin-coloured or pink bump that is translucent. Tiny blood vessels are often visible. BCC often appears on the face and ears. The lesion may rupture, bleed and scab over.

- A flat, scaly, reddish patch with a raised edge often appears on the back or chest.

- A white, waxy, scar-like lesion without a clearly defined border, called morpheaform.

- A brown, blackish lesion with a slightly raised, translucent border.

DNA damage in basal cells results from natural ultraviolet (UV) radiation from the sun and synthetic UV from tanning lamps and beds. UV radiation is 
composed of UVA, UVB and UVC. The most harmful to the human body is UVC. However, UVC is absorbed and reflected by the ozone $\left(\mathrm{O}_{3}\right)$ layer. BCC is reported to be associated with intermittent and childhood sun exposure. UVB is a main causative factor in BCC and has been implicated to directly stimulate DNA mutation via covalent bonding between adjacent pyrimidines, whereas UVA stimulates production of reactive oxygen species that contribute to BCC formation. Factors other than exposure to UV (such as exposure to arsenic, pollutants, viral infections, skin type, age, defects in the immune system) may contribute to the risk and development of BCC. Genetic mutations are important factors in the development of BCC. For example, BCC is a well-recognized cancer, with mutations in the components of the Hedgehog signalling pathway. The p53 tumour suppressor gene is implicated in cancer development and mutation in the p53 gene caused by UVB exposure is relevant in the causation of BCC. Figure 1 displays a general overview of aetiological factors of BCC [1].

\subsection{Treatments}

Several types of treatments are available to remove or destroy BCCs. Selected treatments are dependent on factors such as tumour size, location, a person's age, general health, and preference.

Excision surgery, Mohs surgery, Curettage and electrodesiccation, Radiation therapy, Photodynamic therapy, Chemotherapy, Cryotherapy, Immune response modifiers and Targeted therapy are currently used to treat BCCs.

All of these treatments for BCC have advantages and disadvantages. Other than targeted therapy, all other treatments are indiscriminate, and do not distinguish cancer cells from normal cells. The lack of specificity and selectivity of these treatments usually limit their applications and translate to poor cosmetic outcomes.

The biggest challenges for treatment of $\mathrm{BCC}$ are:

- Education

Understanding BCC causes, risk factors and warning signs may lead to early detection that can be easiest to treat and cure.

- Treatments

The common feature of conventional treatments for BCC is non-specificity without targeting the tumour itself. This leads to unwanted adverse effects in the surrounding tissue, such as scar formation or other cosmetically disfiguring outcomes. Less than favourable recurrence rates are obtained with conventional therapies.

- Depending on the type, location and size as well as preference and ability to do follow-up visits can be daunting for the patient, especially for elderly patients who are also prone to suffer more from this condition.

- An alternative non-invasive, self-treatment modality that is very specific towards the BCC tumour that eliminates small and large BCCs, and is applicable to difficult-to-treat areas with remarkable cosmetic outcomes, resulting in very low recurrence rates, is overdue and welcome. 


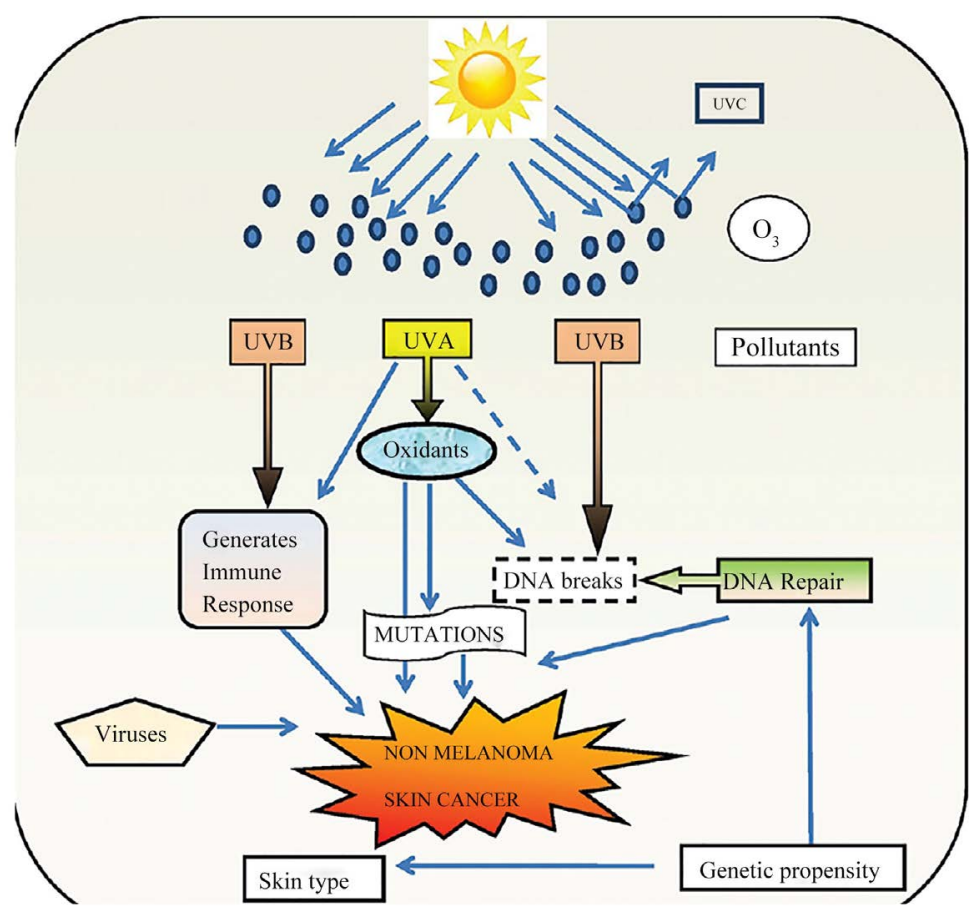

Figure 1. Aetiological factors of basal cell carcinoma (BCC) [1].

\subsection{Curaderm}

In 2018, the product Curaderm was registered by the European Health Authorities as a Medical Device Class 1 for the indication Topical treatment with keratolytic action, and antineoplastic activity in the treatment and healing of localized basal cell carcinoma of the skin. The prime difference between Curaderm and other treatment modalities for BCC is; Curaderm pharmacotherapy discriminates cancer cells from normal cells, which translates to superior efficacy, safety and cosmetic outcomes.

The incidence of BCC is rising, representing approximately $70 \%-80 \%$ of all skin cancers. Accordingly, there is a definite need for more acceptable treatments of BCC as verified by the research into the causes, prevention, and treatment of BCC currently ongoing in many medical research centres throughout the world.

Fundamental studies of BCC have been hindered by a lack of a suitable and reproducible tissue-culture model system. Most available treatments for BCC did not require such models because most BCCs are currently, simply cut out or burnt out and no understanding of mechanism of action at the molecular and cellular levels are required for these indiscriminate procedures.

\subsection{BEC}

In 1987 it was first reported that plant-derived glycoalkaloids solasodine glycosides, including solamargine, solasonine, mono- and di-glycosides of solasodine, known as BEC [2], induced remarkable anticancer effects in cell culture [3], animals [4] and humans [5] [6] [7] [8] [9]. 
Since then, a plethora of further investigations have resulted in the placement of BEC and its individual components as very promising antineoplastic agents with vast potential to serve as targeted anticancer agents [10].

With BEC, solamargine accounts for $86 \%$ antineoplastic activity and solasonine accounts for $9 \%$ antineoplastic activity, whereas, the mono- and di-glycosides of solasodine contribute $5 \%$ anticancer activity. The anticancer activity of these glycoalkaloids is considered to be concerted and additive [11].

The governing principle that determines the potency of antineoplastic activity is the plant sugar rhamnose that forms part of the glycoalkaloids [12]. BEC targets specific mutant proteins on cancer cell membranes that act as specific receptors. After binding to these characterised specific receptors [13] [14] [15] [16] [17], BEC is internalised by cell receptor-mediated endocytosis followed by the anticancer sequelae of identifiable anticancer properties on a variety of biological pathways, including cell survival pathways [18], tumour suppressor pathways [19], lysosomal pathways [20], mitochondrial pathways [21], caspase activation pathways [22], death receptor pathways [23], protein kinase pathways [24] and signal pathways that impede invasion/migration [13] [24] and multidrug resistance [13] [25].

BEC exhibits much higher cytotoxic effects on cancer cells than currently used antineoplastic agents such as vinblastine, vincristine, camptothecin, cisplatin, 5-fluorouracil, gemcitabine, epirubicin, cyclophosphamide, taxol and doxorubicin [26].

Furthermore, the absolute concentrations of these drugs to obtain comparable efficacy as BEC, are in the order of 6 - 40 times higher [27].

Moreover, the therapeutic index (TI: also referred to as the therapeutic ratio) is much higher for BEC compared with other antineoplastic agents as shown with cell culture studies [28] and animal studies [29]. The high TI of BEC translates to high safety margins.

BEC is active against a wide variety of cancer cells, such as ovarian cancer [30], basal cell carcinoma [31], squamous cell carcinoma [32], melanoma [33], colorectal cancer [34], bladder cancer [35], oral epidermoid carcinoma [36], breast cancer [25], leukemia [37], prostate cancer [38], liver cancer [39], lung cancer [40], pancreatic cancer [41], gastric carcinoma [23], renal cancer [31], uterine cancer [30], mesothelioma [42], glioblastoma [43] and osteosarcoma [19]. In addition, BEC has curative properties in animals with terminal cancer [4] [12].

\section{BEC and Basal Cell Carcinoma}

The first human clinical studies with BEC were undertaken in the 1980s in Australia, at the University of Queensland, the Royal Brisbane Hospital, and St. Johns Park Hospital, Tasmania. In 1987, it was reported that topical application of a cream formulation containing BEC was effective in the treatment of the malignant tumour BCC. Histological analyses of biopsies taken before, during and after treatment gave compelling evidence of the efficacy of the formulation. The 
treated lesions did not recur for at least 3 years after cessation of therapy, and indeed, patients were subsequently followed up for over a decade with no recurrences. Thirteen patients with 24 BCC lesions were treated for 4 to 7 weeks. With the BEC therapy, the BCCs responded rapidly in a characteristic pattern. The pattern comprised initial swelling of the lesion, and erythema of surrounding tissue (approx. $10 \mathrm{~mm}$ around the lesion), followed in about 2 days by ulceration until neoplastic cells were destroyed. This was then followed by normal new non-malignant cell growth during the treatment. In the 13 patients treated, 20 of a total number of 24 lesions regressed totally. Partial regression was obtained with the other 4 lesions.

Two normal subjects were treated with the formulation for 8 weeks. Biopsies taken from the treated areas showed no macroscopic or microscopic changes. No clinical reaction was observed with the normal skin. The specificity towards abnormal cells in the skin was the most striking observation.

Biochemical, haematological and urinalytical studies demonstrated that there were no adverse effects on the liver, kidneys or haematopoietic system during or after treatment.

There were no major adverse side effects during BEC therapy except that transient mild itching and burning surrounding the treated lesions occurred in a few cases [5].

Following reports on the anti-cancer effects of BEC in cell culture and in whole animal models, together with the effective treatments for skin cancer, a monograph of BEC was published [44].

In the first human clinical studies, $10 \%(100,000 \mathrm{mg} / \mathrm{L}) \mathrm{BEC}$ was used in a cetomacrogol base to treat the BCCs. In the monograph of BEC, it was reported that patients tolerated up to $50 \%$ BEC in the cetomacrogol cream without any side effects. It was interesting that in cell culture studies, $8 \mathrm{mg} \mathrm{BEC/L}$ cell culture medium was required to kill cancer cells but not harm normal cells. Similarly, 8 $\mathrm{mg} \mathrm{BEC/kg}$ when injected into mice with terminal tumours resulted in survival. Identical doses of BEC injected into normal animals did not produce adverse effects [4].

The doses of BEC to obtain efficacy for the treatment of skin cancer was compared with the doses of BEC required for the treatment of cancers in cell culture and in animals, there was a difference of a factor of over 10,000. It was subsequently shown that this difference was due to the bioavailability of BEC with the cancer cells. Much further work led to a topical formulation that contained keratolytic components that markedly improved the bioavailability of BEC to interact with skin cancer cells. A concentration of BEC, as low as $50 \mathrm{mg} / \mathrm{L}$ of cream, was shown to be effective to treat BCC.

\subsection{Open Studies}

\section{Curaderm and Basal Cell Carcinoma}

Consequently, a clinical study was undertaken to treat 28 patients with 39 BCC lesions with very low concentrations of BEC (5 mg\%), 10\% salicylic acid 
and 5\% urea in a stabilized cetomacrogol cream formulation, Curaderm. Two patients with BCCs were treated with a placebo formulation. In this open study, clinical and histological observations indicated that all 39 BCCs treated with Curaderm had totally regressed. A placebo formulation had no effect on treated lesions. Curaderm had no adverse effect on the liver, kidneys or haematopoietic system. The treatment period to obtain complete regression of the BCC lesions ranged from 3 to 13 weeks. In the placebo group, treatment period was for 14 weeks, which was 1 week more than the longest treatment period with Curaderm [6].

It was remarkable that even at the early developmental stages, Curaderm was able to eliminate BCCs that were almost impossible, due to their locations and sizes, to successfully be treated by other modality treatment procedures.

Photographic documentation of two examples was presented (Figure 2 and Figure 3). Figure 2 is a BCC of the nose, which was present for at least 12 months before treatment started. Independent prognoses for this patient were plastic surgery of limited effect and/or complete loss of the nose, which was to be replaced with a prosthesis.

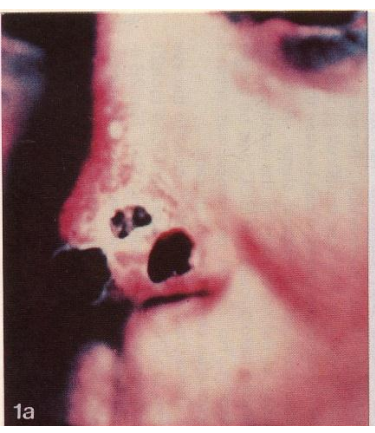

(a)

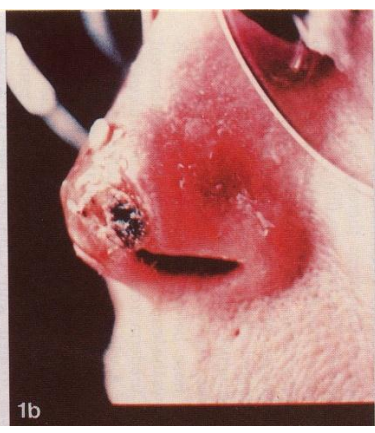

(b)

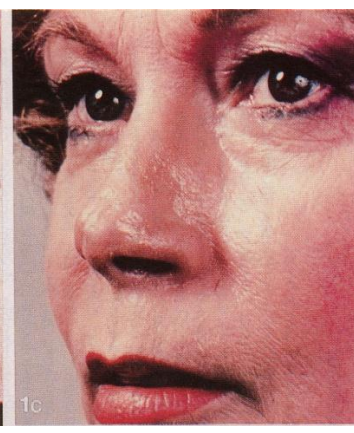

(c)

Figure 2. Clinical diagnosis of a BCC on the nose of a patient before treatment with $\mathrm{Cu}$ raderm (a), during therapy (b), and site of the treated BCC after completion of therapy (c).

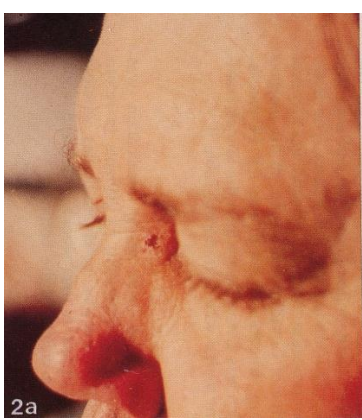

(a)

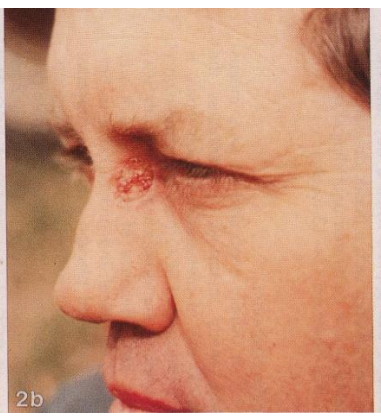

(b)

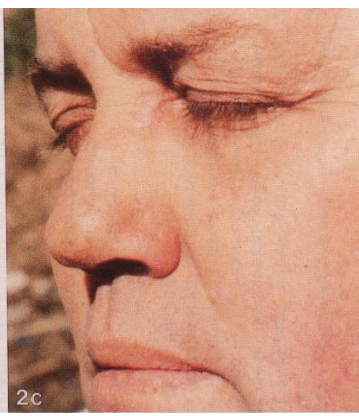

(c)

Figure 3. Clinical progress of a periocular BCC on a patient before treatment (a), two weeks after commencement of therapy (b), and site of treated BCC after completion of therapy with Curaderm (c). Treatment period was 5 weeks. Histological analyses before and after therapy showed that the periocular BCC was cleared with Curaderm therapy. There was no recurrence after 5 years 
Figure 2(a) illustrates several lesions on the nose before treatment started. Figure 2(b) shows the lesion 3 weeks after the commencement of Curaderm pharmacotherapy. The lesions ulcerated over a large area giving the appearance of one large single lesion. During this period the ulceration progressed to the extent that the soft tissue (cartilage) of the nose was visible. Figure 2(c) illustrates where the BCC was. The patient was treated with Curaderm for 13 weeks. A biopsy taken at the conclusion of the treatment showed histologically that BCC was no longer present. No clinical recurrence was seen 10 years after completion of Curaderm pharmacotherapy. This impressive case showed that during treatment, while the cancer cells were dying, new normal skin cells were replacing the dead cancer cells. This confirms the observations experienced with cell culture and animal studies that BEC preferentially attacks and kills cancer cells and leaves normal cells alone.

Another case was presented that could not be successfully treated by other procedures without losing the eye.

Figure 3(a) shows the lesion that was present for 4 months and was growing rapidly. Figure 3 (b) illustrates the lesion 2 weeks after commencement with Curaderm pharmacotherapy. There was a distinct area of ulceration, which was larger in area than the clinically distinguishable BCC prior to the Curaderm treatment. Figure 3(c) shows where the BCC was after treatment with Curaderm. The treatment period in this case was 5 weeks. A biopsy taken at the conclusion of the treatment indicated histologically that BCC was no longer present after treatment with Curaderm. There was no clinical recurrence of the treated BCC for at least 5 years.

The rest is history, many other clinical studies with Curaderm followed, confirming the original observations.

\subsection{Double-Blind, Vehicle-Controlled, Randomized, Paralleled Group Studies}

In the 1990s a double-blind, vehicle-controlled, randomized, paralleled group study to assess the efficacy and safety of BEC-5 (Curaderm) in the treatment of patients with basal cell carcinoma, was carried out by ten centres in the United Kingdom. The study was conducted in full conformance with the principles of the current Declaration of Helsinki. The study was conducted according to International Conference on Harmonisation (ICH) Good Clinical Practice (GCP) guidelines (1996). In 2008 these Phase III studies were published [45].

In these Phase III studies, the treatment groups consisted of 62 patients who were treated for 8 weeks with Zycure, which is Curaderm, and 32 patients who were treated with placebo for 8 weeks. Curaderm pharmacotherapy proved to be a safe therapy for BCC, with a cure rate of $66 \%$ at 8 weeks and $78 \%$ at 1 -year follow-up.

The mode of action was apoptosis of cancer cells with preferential in its application to transformed cells. Patients experienced regeneration of new epider- 
mis at the application site within the first 4 - 5 weeks of treatment, despite twice daily application of Curaderm.

Histological examination showed acanthosis of the regenerated epidermis on an 8-week post treatment biopsy indicating the specificity of Curaderm toward cancer cells and not harming non-cancer cells. Punjabi et al. concluded that Curaderm has overall efficacy, patient acceptance, low incidence of local adverse events and no systemic side-effects [45].

Further studies were recommended to investigate the safety and efficacy of Curaderm with BCCs:

- At different sites of the body

- Different histological types

- More invasive forms

These recommended further studies were addressed by a second trial at the Dermatology Department at the Royal London Hospital, comprising 41 patients, which determined that patients with invasive BCC, such as morpheoic BCC lesions, were successfully treated with Curaderm with a success rate of $78 \%$ when treated for 8 weeks [46].

It was reported that the cosmetic results offered by treatment with Curaderm were comparable to that resulting from surgical excision, but the risks of surgical intervention were well known.

The investigators at the Royal London Hospital further commented that excision of BCC from the facial area involved reconstructive surgery, of which could be both time consuming and costly.

They stated that an alternative, safe, efficacious and cost-effective method of treatment of BCC that did not require physician or hospital attendance should be encouraged, and that Curaderm was a much-needed alternative to surgery for both primary and secondary skin cancer care [46].

It is noteworthy that the trials in the United Kingdom were designed to treat the patients for 8 weeks, which resulted in a $78 \%$ success rate for both superficial and invasive BCCs. In analysing prior and subsequent studies that involved BCC treatments for 14 weeks, it becomes clear that a success rate at 8 weeks treatment was also approximately $80 \%$. Longer treatment periods were required to obtain a higher success rate [27]. These observations complemented the clinical trials.

In order to determine the limitation of topical Curaderm pharmacotherapy, patients with a wider variety of BCCs were studied. In particular, factors indicating poor treatment prognosis in BCC such as size, type, depth, location and previous failure by other treatment modalities, were selected for the study.

\subsection{Curaderm Pharmacotherapy and Size of Basal Cell Carcinoma}

The recurrence rates of large BCCs (over $2 \mathrm{~cm}$ ) are very high, regardless of treatment modality and the cosmetic prognosis is very limited. Curaderm topical pharmacotherapy has resulted in remarkable outcomes with very large BCCs with dimensions of $5 \mathrm{~cm} \times 5 \mathrm{~cm}$ with protrusions of $1.5 \mathrm{~cm}$ from the 
skin [27].

Figure 4 shows a large BCC $(4 \mathrm{~cm} \times 4 \mathrm{~cm} \times 2 \mathrm{~cm})$ before treatment. Dermatologists and surgeons had recommended surgical excision and radiotherapy followed by surgical reconstruction with skin grafting. The patient who had this BCC for at least 3 years elected to treat the lesion with Curaderm. Histological analysis of a biopsy determined that the lesion was a BCC.

This case, as with many other similar cases, shows that regeneration of new epidermis at the application site during treatment of skin cancers with Curaderm occurs, and supports the preclinical and clinical observations that BEC is preferential in its action towards transformed cells. This also explains the impressive clinical and cosmetic outcomes with topical Curaderm pharmacotherapy as shown in Figure 5 and Figure 6 [47] [49].
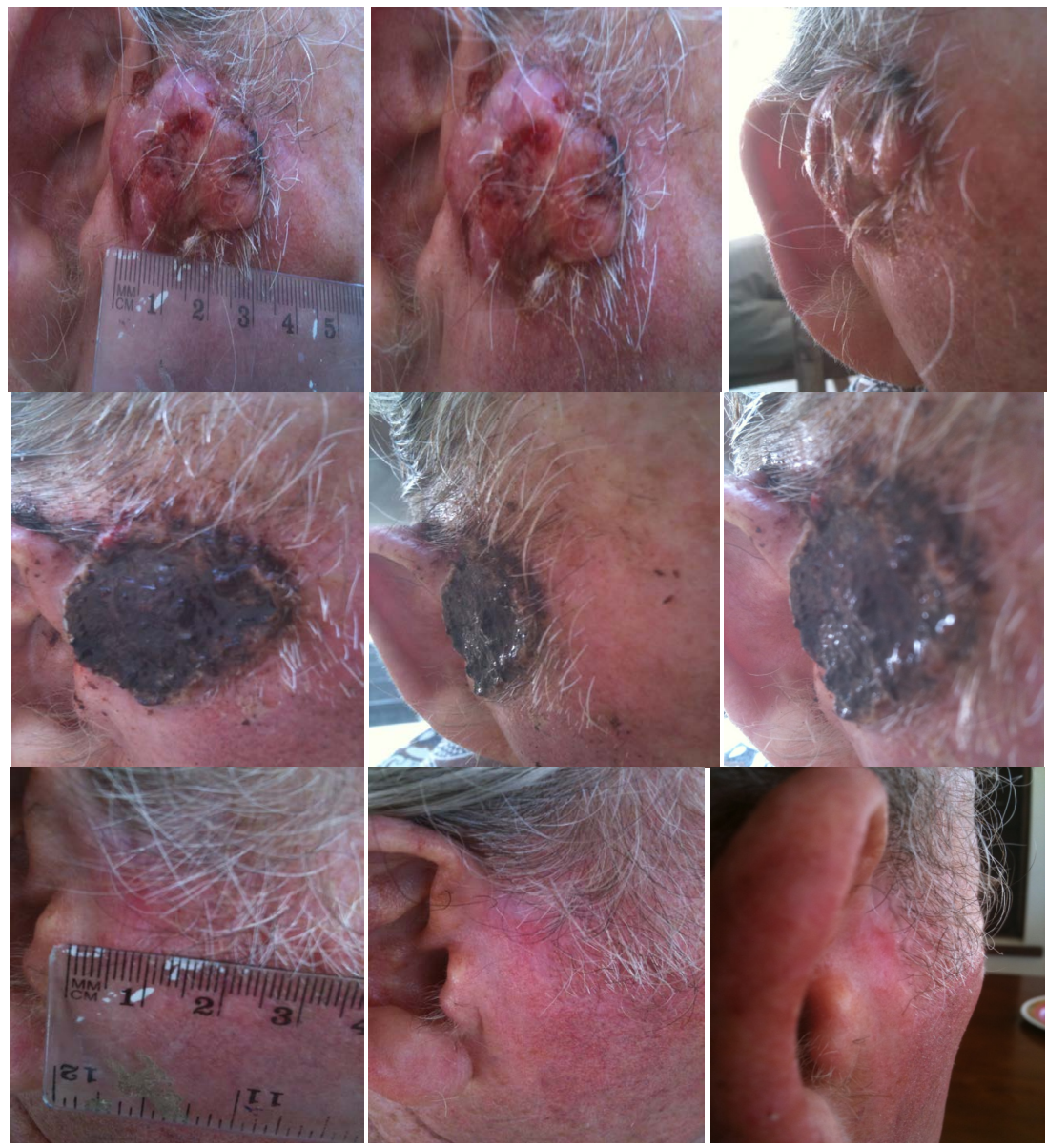

Figure 4. An extensive protruding $(4 \mathrm{~cm} \times 4 \mathrm{~cm} \times 2 \mathrm{~cm})$ BCC with central ulceration and raised curly borders on the right side of his face next to his ear is seen in this patient (top row). Treatment with Curaderm resulted in rapid breakdown of the tumour and after 2 weeks of treatment the lesion was reduced to about a half of the original size. Minor bleeding had occurred during this treatment period (middle row). After 14 weeks of treatment the lesion was clinically eliminated. Normal skin cells had replaced the tumour and the cosmetic end result was excellent, with no scar tissue formation. Even the hairs had regrown where the tumour was originally (bottom row). 


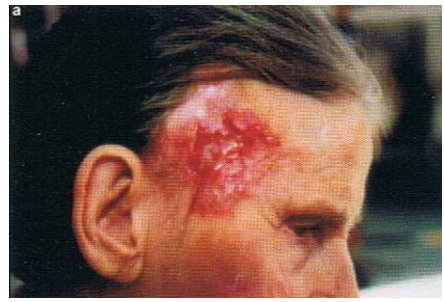

(a)

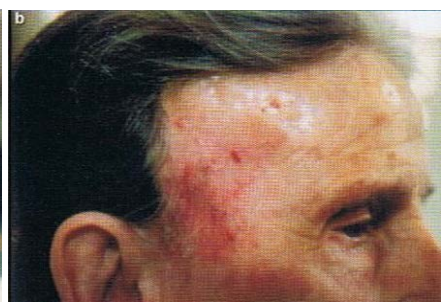

(b)

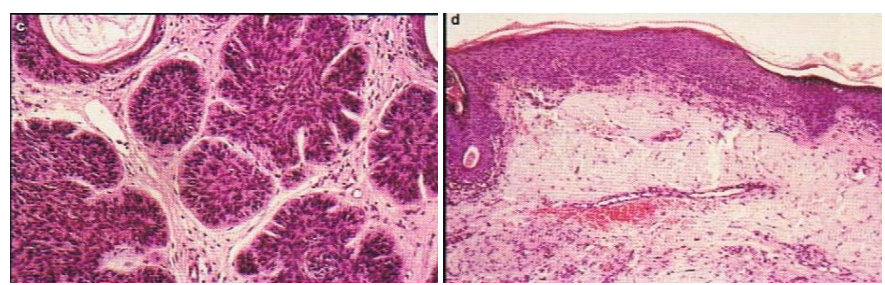

(c)

(d)

Figure 5. Large BCC on the temple of a woman (a). This BCC had been surgically removed and skin grafts applied on two previous occasions only to return. Four weeks treatment with Curaderm resulting in full regression (b). Note the cosmetic result. The clinical diagnosis was confirmed histologically by punch biopsy (c). After completion of the therapy histopathology determined that no residual cancer was present (d) [49].

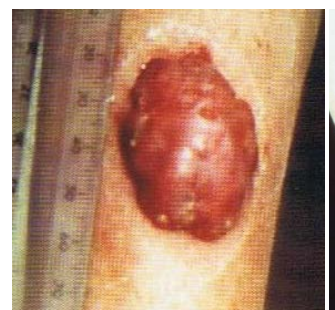

(a)

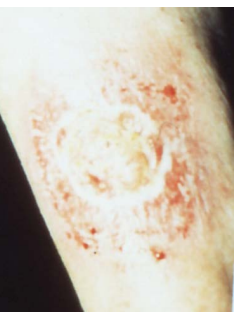

(b)

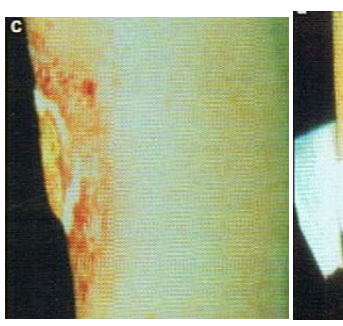

(c)

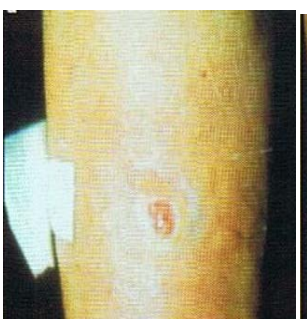

(d)

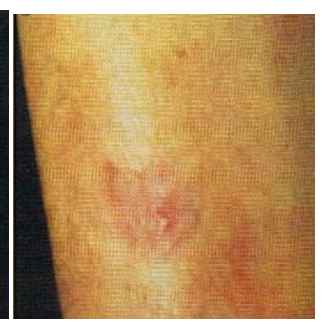

(e)

Figure 6. Large BCC on the leg (a). Note how rapid the cancer was being destroyed by Curaderm during treatment (b-d) and how rapid the wound healed after 5 weeks of $\mathrm{Cu}$ raderm therapy (e). After completion of the therapy histopathology determined that no residual cancer was present. Clinical assessment 5 years post treatment revealed that there was no recurrence [49].

\subsection{Curaderm Pharmacotherapy and Type of Basal Cell Carcinoma}

Superficial, nodular, infiltrative (morpheaform) with differing depths of BCCs have been successfully treated with Curaderm [5] [6] [8] [13] [15] [17] [26] [27] [28] [31] [36] [47]-[59]. 


\subsection{Curaderm Pharmacotherapy and Difficult to Treat Locations of Basal Cell Carcinoma}

Location of BCCs largely determines, and, limits the choice of treatment. Curaderm topical pharmacotherapy was shown to excel in these situations.

Figures 7-9 illustrate sensitive areas that have been successfully treated with Curaderm, highlighting one of its main attributes, remarkable cosmetic outcomes [5] [6] [8] [15] [26] [49] [51] [54] [63].

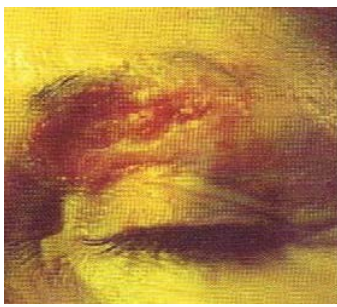

(a)

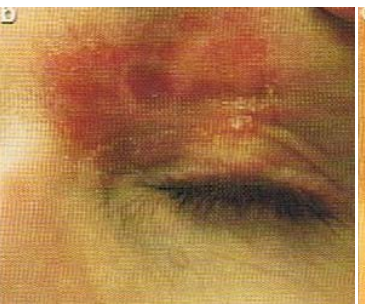

(b)

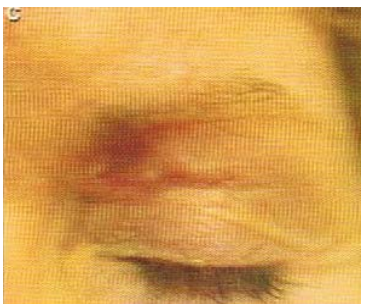

(c)

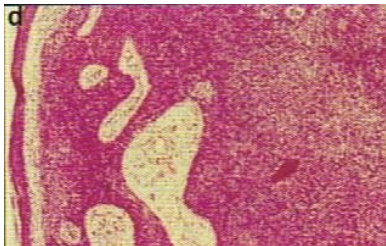

(d)

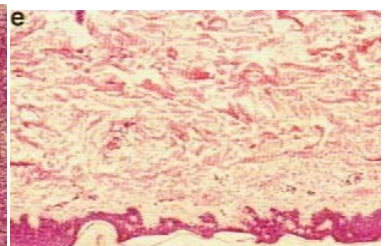

(e)

Figure 7. Periocular BCC, superior to the eye including part of the brow, of a patient before (a), during (b) and after (c) Curaderm therapy. During Curaderm treatment the lesion was much smaller and residual tumour can distinctly be seen which was surrounded by some inflammation. After treatment there was no sign of the BCC. Confirmation by histological analysis of the BCC before treatment (d), and after treatment (e) are shown. The total treatment period was 9 weeks. Clinical assessment 5 years post treatment showed that there was no recurrence [49].

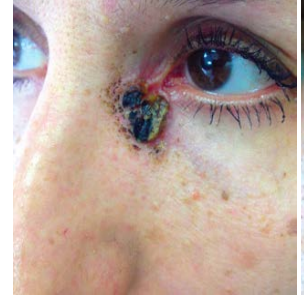

(a)

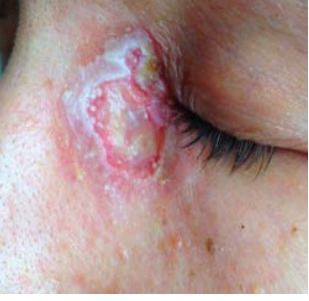

(b)

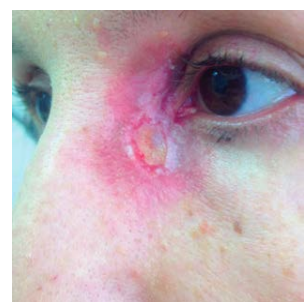

(c)

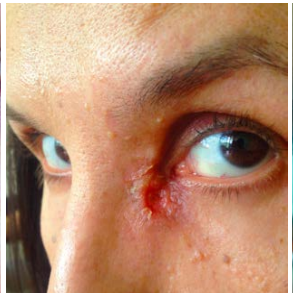

(d)

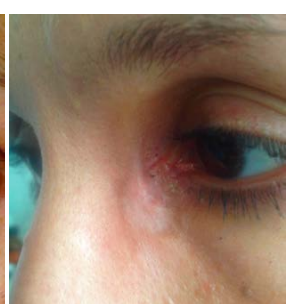

(e)

Figure 8. Patient with a BCC dangerously close to left eye, before Curaderm pharmacotherapy (a), during different stages of therapy (b-d), after 8 weeks treatment (e). 


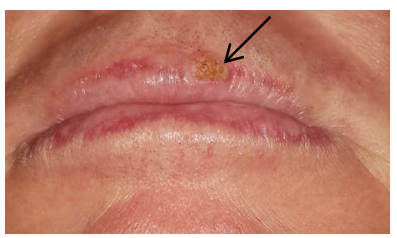

(a)

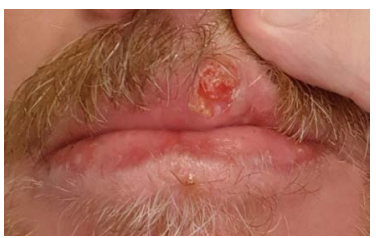

(b)

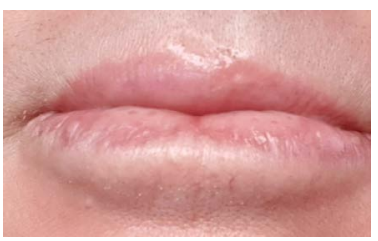

(c)

Figure 9. BCC on upper lip [see arrow] that had been troubling a 53-year patient for some time, before Curaderm therapy (a), during (b) and after (c) Curaderm therapy.

\subsection{Curaderm Pharmacotherapy on Previously Failed Treatment Procedures}

A number of treatments are available for topical BCCs with varying success and recurrence rates. When a $\mathrm{BCC}$ recurs after treatment with virtually any procedure, scar tissue poses a problem for retreating the lesion. Curaderm pharmacotherapy was evaluated for its application under such circumstances.

Twenty-three patients that had a recurrence by other modality treatments such as radiation therapy, photodynamic therapy, laser therapy, cryotherapy and then subsequently treated with Curaderm, resulted in $52 \%$ success rate with no recurrences with 5 years follow up [60].

Figures 10-12 illustrate various lesions that had recurred after treatment with radiation therapy, photodynamic therapy and laser therapy and then treated successfully with Curaderm [56].

Figure 13 shows a large lesion on the nose of a patient who had previous treatments with surgery, radiation and photodynamic therapy. On all occasions of the treatments, the lesion had recurred. Topical Curaderm pharmacotherapy of the same multiple recurred lesion successfully removed the lesion with no recurrence for over 3 years [61].

\subsection{Curaderm and Elderly Patients}

There is a correlation between the incidence of BCC and increasing age in humans. Clinicians are usually the frontline to encounter elderly patients with BCCs at their general check-up visits. Once the diagnosis of the BCC is made, both the clinician and the patient are faced with the challenge of treatment. All treatment modalities have special considerations when administered to older adults. The physical condition and ability of the patient and the characteristics of the BCC tumour type and locality may limit the choices of treatment. Dobrokhotova et al. [62] have considered the need of an appropriate treatment procedure for elderly patients. These investigators have appreciated that the elderly encounter restrictions when treatment is required for their BCC, and have 
evaluated Curaderm as a possible candidate and solution for this large group of patients. In particular, they selected patients with lesions at locations that are considered difficult to treat such as, on the head and neck.

In 2016, they published their findings in the journal "Tumors of the Head and Neck" [62]. They concluded that Curaderm is a successful treatment of BCC of the head and neck. Figure 14 and Figure 15 illustrate two elderly patients suffering with BCCs that have successfully been treated with Curaderm and their impressive cosmetic results after treatment. These observations contribute value to the patient and Health Care Providers.

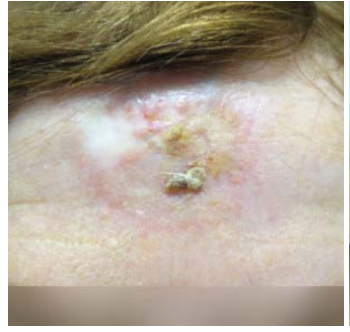

(a)

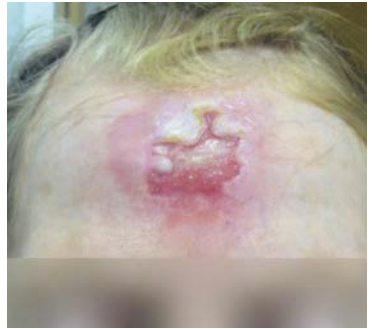

(c)

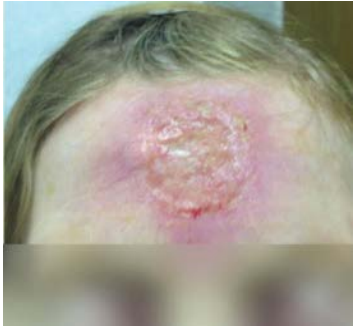

(b)

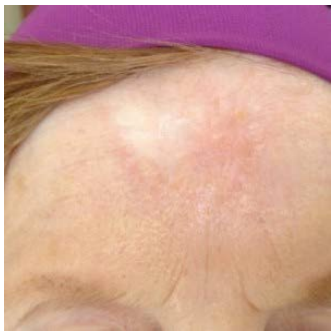

(d)

Figure 10. Recurrent BCC after radiation therapy but before Curaderm therapy (a), during treatment with Curaderm (b, c) and after 8 weeks treatment (d).

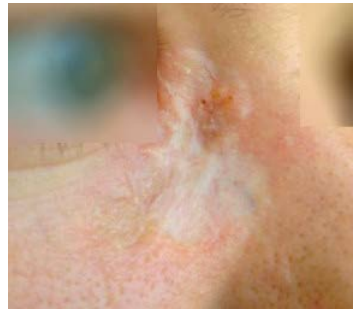

(a)

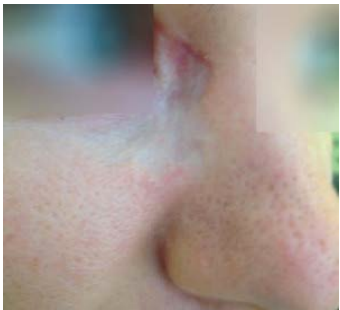

(c)

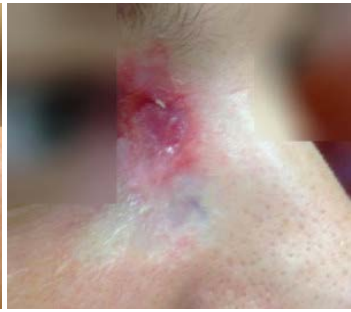

(b)

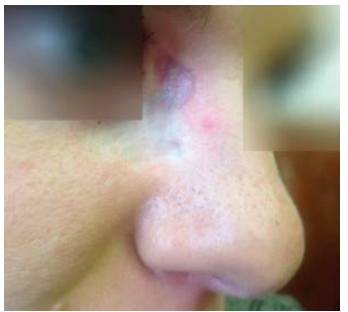

(d)

Figure 11. Recurrent BCC close to the eye after laser therapy but before Curaderm therapy (a), during treatment with Curaderm (b, c), and after 8 weeks treatment (d). 


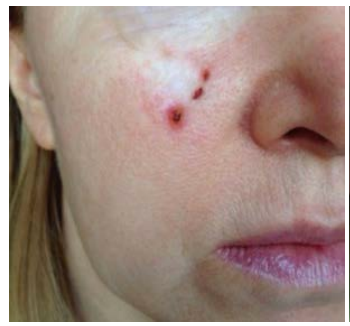

(a)

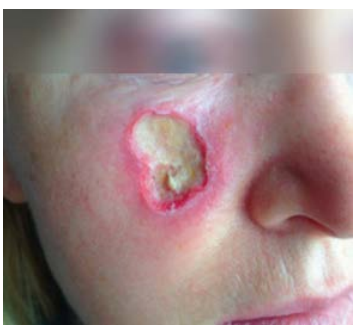

(b)

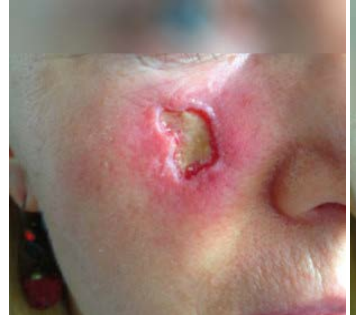

(c)

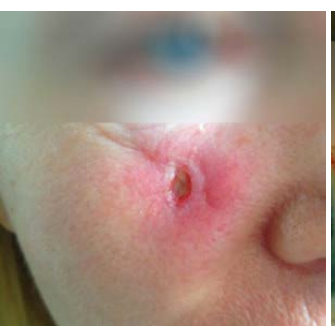

(d)

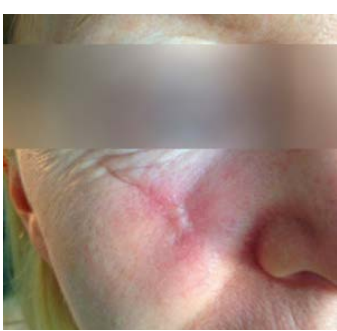

(e)

Figure 12. Relapsed BCC on cheek after PDT but before Curaderm therapy (a), during Curaderm treatment (b, c, d) and after 10 weeks of treatment (e).

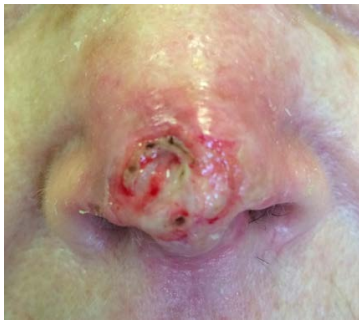

(a)

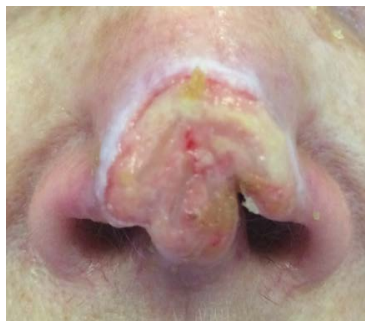

(c)

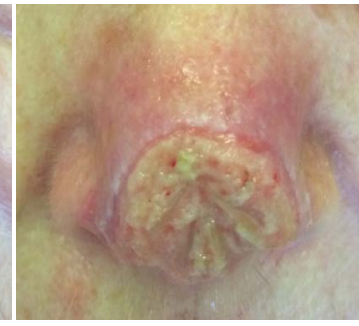

(b)

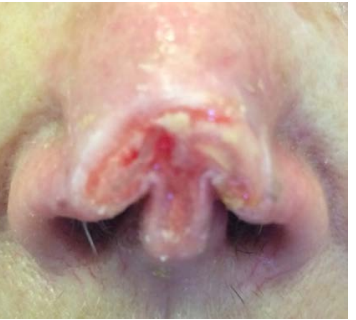

(d)

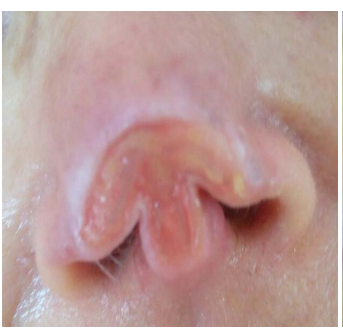

(e)

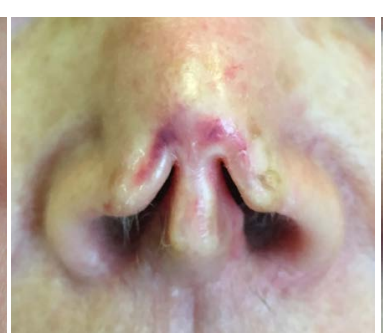

(f)

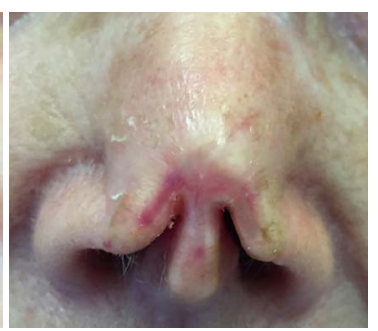

(g)

Figure 13. A large infiltrating refractory BCC, which recurred after three failed attempts by widely accepted therapies; (a) immediately before commencement with Curaderm therapy; (b) 2 weeks; (c) 5 weeks; (d) 8 weeks and (e) 14 weeks during Curaderm therapy. Treatment stopped at 14 weeks of therapy. Appearances of treated lesions (f) 7 weeks and (g) 10 weeks after cessation of treatment. 


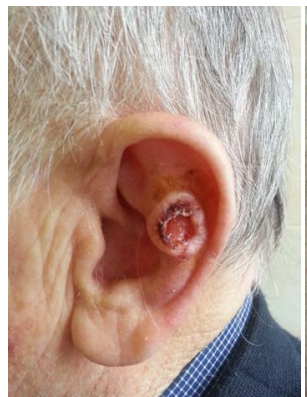

(a)

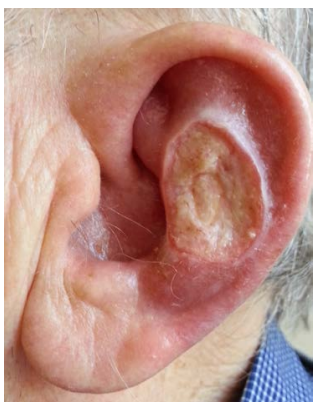

(d)

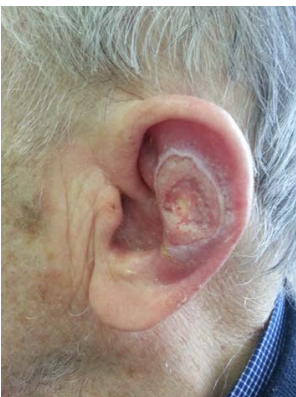

(b)

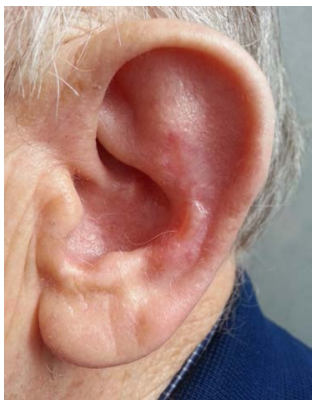

(e)

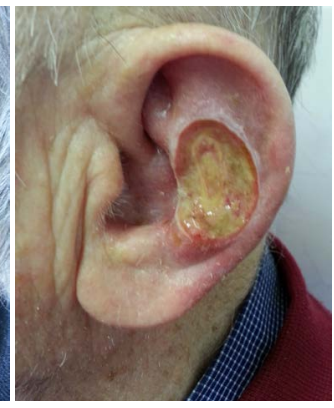

(c)

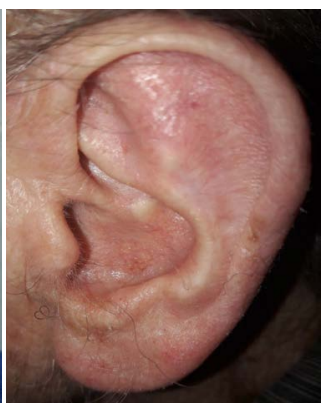

(f)

Figure 14. Patient, 85 years. BCC on left ear, before Curaderm pharmacotherapy (a), after 17 days treatment (b), after 28 days treatment (c), after 60 days treatment (d), after 74 days treatment (e). No relapse after 5 years treatment (f).

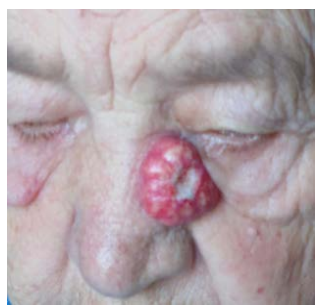

(a)

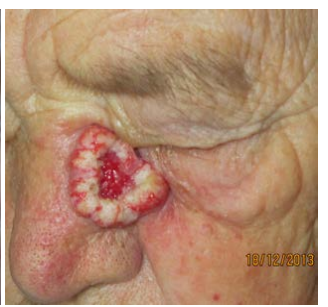

(b)

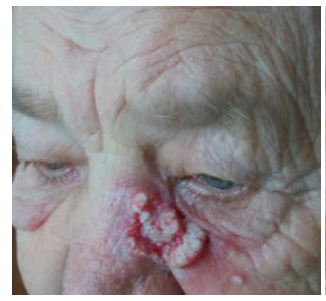

(c)

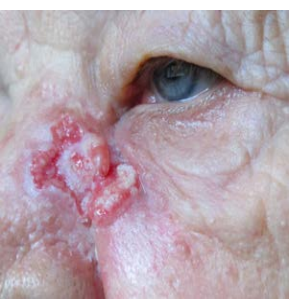

(d)

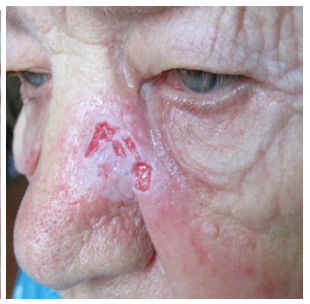

(e)

Figure 15. Patient, 91 years. BCC close to the left eye, before Curaderm pharmacotherapy (a), during different stages of therapy (b-d), after 14 weeks treatment (e).

\subsection{Other Clinically Relevant Studies with Curaderm and Basal Cell Carcinoma}

A study showing graphic and pictorial images what occurs clinically during Curaderm pharmacotherapy has been reported in 2015 [55].

The clinical observations with Curaderm pharmacotherapy reveal that initially, the BCC lesion size increases over four-fold due to the interaction of 
Curaderm with deeper and more lateral tumour cells, followed by a decrease in size, ultimately resulting in complete elimination of the BCC. Figure 16 shows the changes in areas of the $\mathrm{BCC}$ lesion relative to pre-treatment area vs. treatment times, and beyond treatment times. The lesion responded rapidly to the treatment. There was an immediate increase in lesion size after commencement of Curaderm pharmacotherapy. The size of the lesion increased more than four-fold and peaked at approximate 30 days of treatment. On-going treatment with Curaderm then resulted in a decline of lesion size, at day 59 the size had returned to the original pre-treatment size. Continuing treatment caused the lesion to further reduce in size and after 86 days of treatment the lesion was completely eliminated. From approximately day 30 treatment, regeneration of new epidermis at the application site occurred until the end of therapy (day 86) despite continued three times daily application of Curaderm.

Figure 17 illustrates the photographs that correspond to the events described for Figure 16.

The sequence of these observations always occurs when treating BCCs with Curaderm.

The extent of the changes in size of the lesions and the duration of these changes during treatment depend on the original pre-treatment size and characteristic of the BCC.

Very importantly, as was shown in all cases that were treated with Curaderm pharmacotherapy, whilst Curaderm is destroying cancer cells, normal cells are replenishing the dead cancer cells and this exceptional occurrence translates to the observed impressive cosmetic outcomes. There are no other treatments available that render this selectivity.

All other available treatments are indiscriminate with consequential cosmetic limitation outcomes.

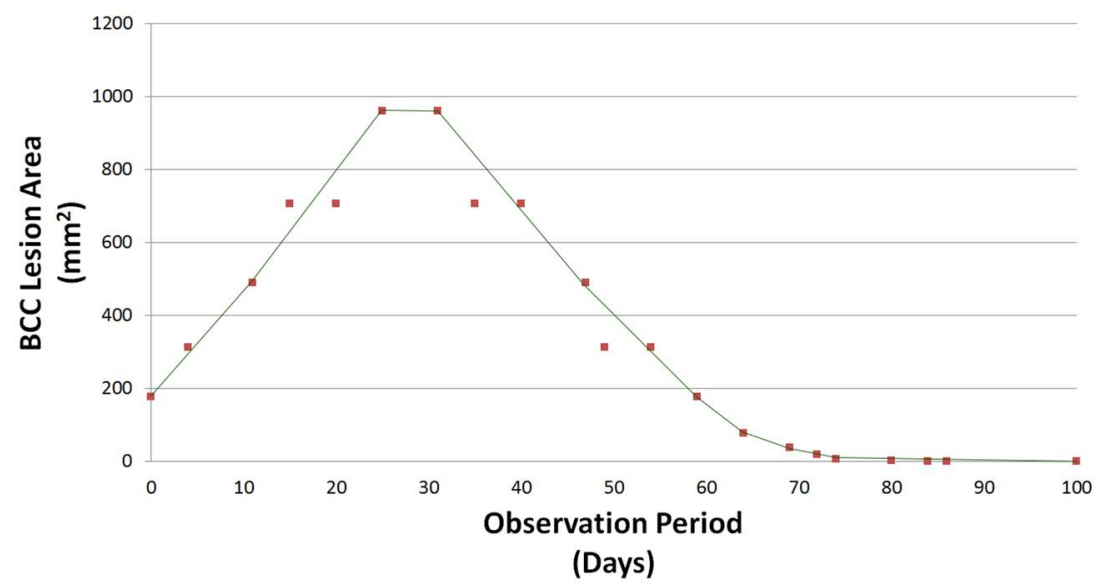

Figure 16. Curaderm therapy caused an immediate change in BCC lesion size and after 30 days treatment, peaked at over a 4 -fold increase in size. Continuous treatment after 30 days resulted in a decrease in lesion size and complete removal of the BCC was attained after 86 days of Curaderm therapy. Before treatment started the diameter of the lesion was $15 \mathrm{~mm}\left(177 \mathrm{~mm}^{2}\right)$ [55]. 


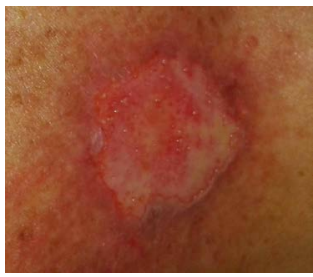

Day 4

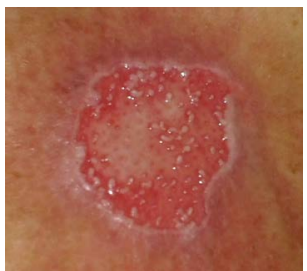

Day 25

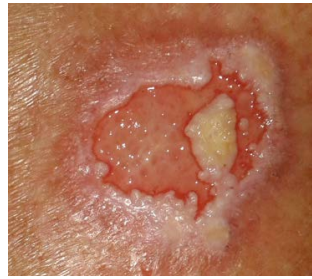

Day 40

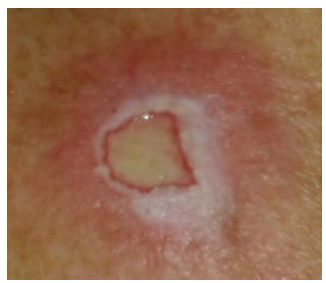

Day 54

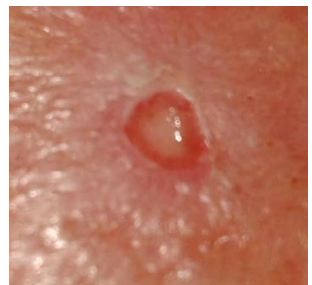

Day 69

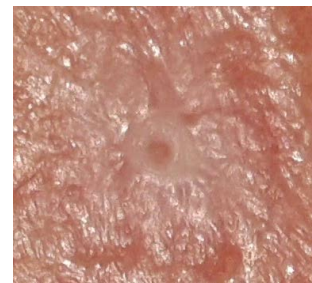

Day 84

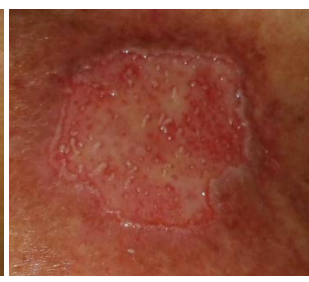

Day 11

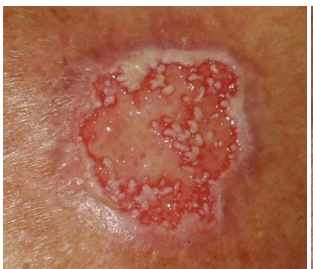

Day 30

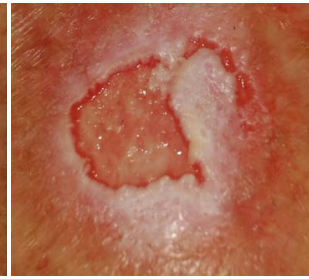

Day 47

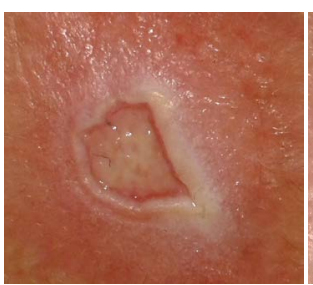

Day 59

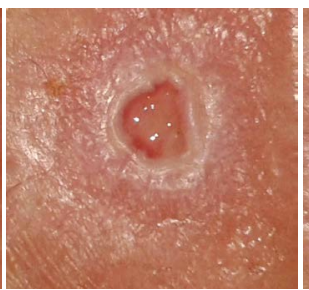

Day 72

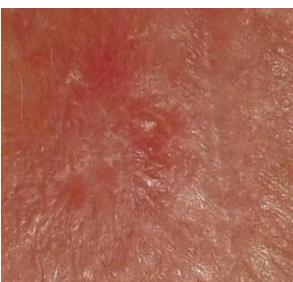

Day 86

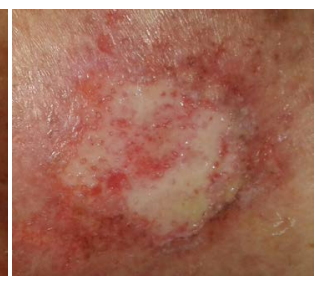

Day 15

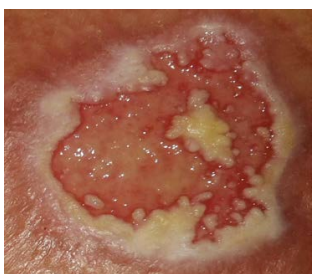

Day 35

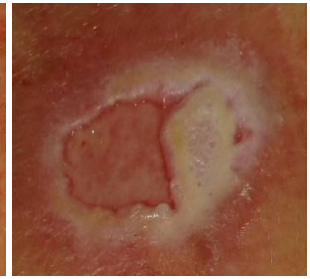

Day 49

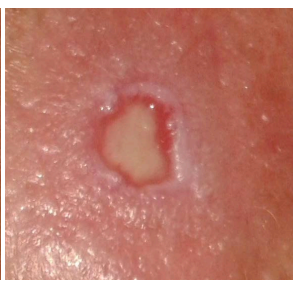

Day 64

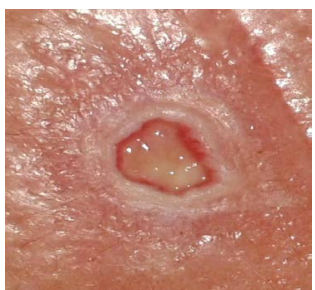

Day 74

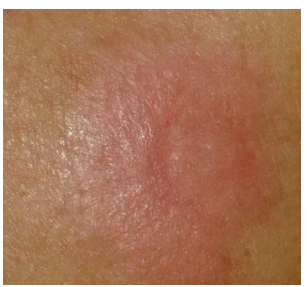

Day 100

Figure 17. Appearances of BCC lesion during and after Curaderm therapy. Curaderm was applied 3 times daily at a dose of $0.1 \mathrm{~g}$ cream and covered with micropore paper tape occlusive dressing. The indicated days refer to the treatment periods. Cancer cells were being eliminated and replaced with normal epidermal skin cells during treatment. Treatment was stopped only after the original BCC lesion had healed (day 86). 


\subsection{Compliance and Tolerance}

Many studies attest to good compliance and tolerance when on Curaderm pharmacotherapy. The only reported side effects were mild erythema with transient burning sensations at the treatment site. These sensations were dependent on the sizes of the lesions. Nevertheless, the treatment was well tolerated by all patients [63], confirming many other studies [5] [6] [11] [26] [27] [31] [48] [49] [51] [52] [53] [55] [56]-[62].

\subsection{Population Studies with Curaderm}

Curaderm pharmacotherapy has been used in different populations and ethnicity throughout the world with similar outcomes. For example, in the Russian Federation, 95 patients were treated with Curaderm at the Russian Cancer Research Centre of Russian Academy of Medical Sciences. The duration of treatment with Curaderm ranged from 4 weeks to 14 weeks. Seventy-two patients with primary BCC when treated with Curaderm resulted in $82 \%$ success rate. The cosmetic outcomes after treatment with Curaderm were remarkable [60].

\subsection{Rare and Long-Term Adverse Effects of Curaderm}

Phase IV studies with Curaderm were designed to detect any rare or long-term adverse effects over a much larger patient population and timescale than was possible during the initial clinical trials. Curaderm has been available and used for over two decades and only a very small number of patients have experienced minor scar tissue caused by the salicylic acid in the Curaderm cream. The reasons for the scar tissue were applications of too much cream to the site, sensitivity to salicylic acid, and application to non-indicated conditions such as dry sensitive skin. These observations have secured Curaderm pharmacotherapy as having exceptional effective and safety profiles.

\subsection{Coping with BCC Treatment, Does Curaderm Pharmacotherapy Assist?}

There are different types of side effects, usually not reported, that patients endure when diagnosed and treated for BCC.

Side effects such as emotional, physical and financial cost of BCC can be very high depending on mainly, size and location of the lesion.

Emotional and social effects, as well as physical effects, may include sadness, anxiety, anger or not being able to control stress levels. Not knowing the cosmetic outcome of the selected treatment procedure, places tremendous stress on the patient and if the cosmetic outcome of their treatment is not to their satisfaction, the patient has to live with this trauma from day to day, and the patient may lose confidence in the medical profession.

\subsection{The Financial Stress Can Have a Significant Bearing on the Patient}

BCC treatment can be expensive. It is often a big source of stress and anxiety for 
people with BCC and their families. In addition to treatment costs, there are possibly extra, unplanned expenses related to their care. High cost of medical care may result in the patient stopping or not completing the treatment. This can put the health of the patient at risk and may lead to higher cost in the future.

The many advantages of having available self-treatments under the supervision of a health care professional are significant for both the patient and for the Health Care Professional. The safety and treatment outcomes are the prime considerations for the patient. Curaderm has proven to be very safe and the cosmetic outcome is impressive and is similar, if not better, than surgery, which may include reconstruction and grafting.

The lower cost of the treatment with Curaderm, compared to established therapies, is beneficial for the patient and for the Health Care System. Any modality that can reduce the already financial burden to the Health Care System and to patients should be considered seriously.

\subsection{Limitation of Curaderm Pharmacotherapy}

The main limitation of topical pharmacotherapy with Curaderm is the duration of treatment, which depends on the type and size of the BCC and may take several weeks up to several months therapy for the complete removal of the BCC. Transient stinging and burning sensations are experienced in some patients for several minutes after application of Curaderm cream to the lesions. However, these limitations are considered minor when compared with the efficacy and impressive cosmetic outcomes, especially at difficult to treat locations.

\section{Conclusions}

The conventional treatments for BCC are surgical excision, Mohs micrographic surgery, curettage and electrodesiccation, chemotherapy, radiation, cryotherapy or laser therapy. The common feature of all of these treatments is that they are nonspecific without targeting the tumor itself. This leads to unwanted adverse effects in the surrounding tissue such as scar formation or other cosmetically disfiguring outcomes. In addition, treatment successes and morbidity of these treatments are questionable and mostly depended on the skill of the operator of the procedure. Hence, an alternative treatment modality that is very specific towards the BCC tumour that eliminates small BCCs rapidly, and also eliminates large BCCs, and difficult-to-treat areas that contain BCCs with amazing cosmetic outcomes, is overdue and welcome.

This astonishing BCC treatment has been achieved by extensive investigations of the natural compound BEC that has been shown invariably to suppress and reverse the cancer-causing biochemical changes. This has led to Curaderm, a pharmacological intervention for cutaneous BCC.

Self-administered, non-invasive topical Curaderm pharmacotherapy offers innumerable benefits unavailable with conventional therapies. Curaderm pharmacotherapy is preferable to invasive procedures, especially in cases of multifocal lesions, unclear lesion edges, risk of hypertrophic scarring and/or keloids re- 
sulting in disfiguring scars, surgical risk factors and localization in some areas such as the face. Molecular targeting therapy with BEC for the treatment of BCC fills the gap of conventional therapies.

Finally, the adage, "prevention is better than cure" is very applicable to BCC.

There are many known risk factors that contribute to the cause of BCC. Many of the known risk factors, if taken into consideration when developing preventative measures, should result in fewer required treatments. Since solar radiation is a major environmental cause of BCC, a combination of sun protection measures should be encouraged. With the advanced understanding of the science of BCC and its aetiology, novel approaches should be motivated to prevent this disease from occurring. To that end, research has shown that BEC in combination with sunscreen agents in a formulation Curasol eliminates very early stages of skin cancer. This preventative manner of approach to influence the fight against BCC should be stimulated.

BCC is the most common form of skin cancer and the most frequently occurring form of all cancers. If allowed to grow, BCC can become disfiguring and dangerous. Neglected BCCs can become locally invasive, grow broad and deep into the skin and destroy skin, tissue and bone. It is therefore important to treat BCC as early as possible to avoid serious repercussions. There are various treatments available. The treatment choice depends on size, location, age of the BCC, age of the patient and the patient's overall health.

The most widely used treatments are all indiscriminate, these treatments do not distinguish cancer cells from normal cells and the ultimate effective and cosmetic success of the treatment relies on the skill of the treating doctor.

On the other hand, Curaderm pharmacotherapy distinguishes cancer cells from normal cells and only eliminates cancer cells at the molecular and cellular levels, resulting in impressive cosmetic outcomes as verified by Phases I to IV clinical studies and open field observations. The recurrence rate of BCC treated with Curaderm is minimal compared with other treatment modalities.

Curaderm pharmacotherapy has been approved in 2018 by the European Health Authorities as a Class 1 Medical Device for the indication "Topical Treatment with Keratolytic Action, and Antineoplastic Activity in the Treatment and Healing of Localized Basal Cell Carcinoma of the Skin".

Curaderm pharmacotherapy offers the long-awaited breakthrough for the treatment of skin cancer, in particular BCC.

\section{Conflicts of Interest}

The authors declare no conflicts of interest regarding the publication of this paper.

\section{References}

[1] Bhandari, P.R. and Pai, V.V. (2014) Novel Medical Strategies Combating Nonmelanoma Skin Cancer. Indian Journal of Dermatology, 59, 531-546. https://doi.org/10.4103/0019-5154.143503 
[2] Cham, B.E. and Wilson, L. (1987) HPLC of Glycoalkaloids from Solanum Sodomaeum. Planta Medica, 1, 59-62. https://doi.org/10.1055/s-2006-962621

[3] Daunter, B. and Cham, B.E. (1990) Solasodine Glycosides. In Vitro Preferential Cytotoxicity for Human Cancer Cells. Cancer Letters, 55, 209-220. https://doi.org/10.1016/0304-3835(90)90121-D

[4] Cham, B.E., Gilliver, M. and Wilson, L. (1987) Antitumour Effects of Glycoalkaloids Isolated from Solanum Sodomaeum. Planta Medica, 1, 34-36. https://doi.org/10.1055/s-2006-962612

[5] Cham, B.E. and Meares, H.M. (1987) Glycoalkaloids from Solanum sodomaeum L. Are Effective in the Treatment of Skin Cancers in Man. Cancer Letters, 36, 111-118. https://doi.org/10.1016/0304-3835(87)90081-4

[6] Cham, B.E., Daunter, B. and Evans, R. (1991) Topical Treatment of Malignant and Premalignant Skin Cancers by Very Low Concentrations of a Standard Mixture of Solasodine Glycosides. Cancer Letters, 59, 183-192. https://doi.org/10.1016/0304-3835(91)90140-D

[7] Punjabi, S., Cook, I., Kersey, P., Marks, R., Finlay, A., Sharpe, G. and Cerio, R. (2000) A Double Blind, Multi-Centre Parallel Group Study of BEC-5 Cream in Basal Cell Carcinoma. Journal of the European Academy of Dermatology and Venereology, 14, 47-60.

[8] Cham, B.E. (2014) A Review of Solasodine Rhamnosides Therapy for in Situ Squamous Cell Carcinoma on the Penis. British Journal of Medicine and Medical Research, 4, 621-631.

[9] Goldberg, L.H., Landau, J.M., Moody, M.N. and Vergilis-Kalner, I.J. (2011) Treatment of Bowen's Disease on the Penis with Low Concentrations of a Standard Mixture of Solasodine Glycosides and Liquid Nitrogen. Dermatologic Surgery, 37, 858-861. https://doi.org/10.1111/j.1524-4725.2011.02014.x

[10] Cham, B.E. (2020) Combination Treatment with BEC and Cisplatin Synergistically Augments Anticancer Activity and Results in Increased Absolute Survival. Journal of Cancer Therapy, 11, 470-482. https://doi.org/10.4236/jct.2020.118040

[11] Cham, A., Cham, K., Chase, T. and Cham, B.E. (2015) A Standardized Plant Extract Containing a Target Compound Is Acceptable as a Potent Therapeutic Entity: Relevance to BEC and Solamargine, a Topical Clinical Formulation CuradermBEC5. Journal of Cancer Treatment and Research, 3, 22-27. https://doi.org/10.11648/j.jctr.20150302.12

[12] Chang, L.C., Tsai, T.R., Wang, J.J., Lin, C.N. and Kuo, K.W. (1998) The Rhamnose Moiety of Solamargine Plays a Crucial Role in Triggering Cell Death by Apoptosis. Biochemical and Biophysical Research Communications, 242, 21-25.

[13] Cham, B.E. (2017) Solasodine, Solamargine and Mixtures of Solasodine Rhamnosides: Pathway to Expansive Clinical Anticancer Therapies. International Journal of Clinical Medicine, 8, 692-713. https://doi.org/10.4236/ijcm.2017.812064

[14] Lipscombe, R., Carter, S. and Ruane, M. (2005) Rhamnose Binding Protein. US Patent No. 6930171.

[15] Cham. B.E. (2007) Solasodine Rhamnosyl Glycosides Specifically Bind Cancer Cell Receptors and Induce Apoptosis and Necrosis. Treatment for Skin Cancer and Hope for Internal Cancers. Research Journal of Biological Sciences, 2, 503-514.

[16] Wang, Y., Gao. J., Gu, G., Li, G., Cui, C., Sun, B. and Lou, H.X. (2007) In Situ RBL Receptor Visualisation and Its Mediated Anticancer Activity for Solasodine Rhamnosides. ChemBioChem, 12, 2418-2420. https://doi.org/10.1002/cbic.201100551 
[17] Cham, B.E. (1994) Solasodine Glycosides as Anticancer Agents: Preclinical and Clinical Studies. Asia Pacific Journal of Pharmacology, 9, 113-118.

[18] An, W.X., Lai, H.L., Zhang, Y.Y., Liu, M.H., Lin, X.K. and Cao, S.S. (2019) Apoptotic Pathway as the Therapeutic Target for Anticancer Traditional Chinese Medicines. Frontiers in Pharmacology, 10, 758. https://doi.org/10.3389/fphar.2019.00758

[19] Li, X., Zhao, Y., Wu, W.K., Liu, S., Cui, M. and Lou, H. (2011) Solamargine Induces Apoptosis with P53 Transcription-Dependent and Transcription-Independent Pathways in Human Osteosarcoma U2OS Cells. Life Sciences, 88, 314-321. https://doi.org/10.1016/j.lfs.2010.12.006

[20] Al Sinani, S.S., Eltayeb, E.A., Coomber, B.L. and Adham, S.A. (2016) Solamargine Triggers Cellular Necrosis Selectively in Different Types of Human Melanoma Cancer Cells through Extrinsic Lysosomal Mitochondrial Death Pathways. Cancer Cell International, 16, 287-294. https://doi.org/10.1186/s12935-016-0287-4

[21] Zang, X.H., Yan, Z.P., Xu, T.T., An, Z.T., Chen, W.Z., Wang, X.S., Huang, M.M. and Zhu, F.S. (2018) SM Derived from Solanum Nigrum Induces Apoptosis of $\mathrm{Hu}$ man Cholangio Carcinoma QBC939 Cells. Oncology Letters, 15, 6329-6335. https://doi.org/10.3892/ol.2018.8171

[22] Al Sinani, S.S.S. and Eltayeb, E.A. (2017) The Steroidal Glycoalkaloids Solamargine and Solasonine in Solanum Plants. South African Journal of Botany, 112, 253-269. https://doi.org/10.1016/j.sajb.2017.06.002

[23] Kalalinia, F. and Korimi-Sani, I. (2017) Anticancer Properties of Solamargine: A Systematic Review. Phytotherapy Research, 31, 858-870. https://doi.org/10.1002/ptr.5809

[24] Sani, I.K., Marashi, S.H. and Kalalinia, F. (2015) Solamargine Inhibits Migration and Invasion of Human Hepatocellular Carcinoma Cells through Down-Regulation of Matrix Metalloproteinases 2 and 9 Expression and Activity. Toxicology in Vitro, 29, 893-900. https://doi.org/10.1016/j.tiv.2015.03.012

[25] Li, X., Zhao, Y., Liu, S.S., Cui, M. and Lou, H.X. (2011) Induction of Actin Disruption and Downregulation of P-Glycoprotein Expression of Solamargine in Multidrug-Resistant K562/A02 Cells. Chinese Medical Journal, 124, 2038-2044.

[26] Cham, B.E. and Chase, T.R. (2012) Solasodine Rhamnosyl Glycosides Cause Apoptosis in Cancer Cells. Do They Also Prime The Immune System Resulting in Long-Term Protection against Cancer? Planta Medica, 78, 349-353.

[27] Cham, B.E. (2013) Inspired by Nature, Proven by Science. The New Generation Cancer Treatment That Causes Cancer Cells to Commit Suicide. Colorite Graphics, Port Vila, 264 p.

[28] Cham, B.E. (2007) Solasodine Rhamnosyl Glycosides Specifically Bind Cancer Cell Receptors and Induce Apoptosis and Necrosis. Treatment for Skin Cancer and Hope for Internal Cancers. Research Journal of Biological Sciences, 2, 503-514.

[29] Cham, B.E. and Daunter, B. (1990) Solasodine Glycosides. Selective Cytotoxicity for Cancer Cells and Inhibition of Cytotoxicity by Rhamnose in Mice with Sarcoma 180. Cancer Letters, 55, 221-225. https://doi.org/10.1016/0304-3835(90)90122-E

[30] Wu, Y.H., Chiu, W.T., Young, M.J., Chang, T.H., Huang, Y.F. and Chou, C.Y. (2015) Solanum incanum Extract down Regulates Aldehyde Dehydrogenase 1-Medicated Stemness and Inhibits Tumor Formation in Ovarian Cancer Cells. Journal of Cancer, 6, 1011-1019. https://doi.org/10.7150/jca.12738

[31] Cham, B.E. (2013) Drug Therapy: Solamargine and Other Solasodine Rhamnosyl Glycosides as Anticancer Agents. Modern Chemotherapy, 2, 33-49. https://doi.org/10.4236/mc.2013.22005 
[32] Wu, C.H., Liang, C.H., Shiu, L.Y., Chang, L.C., Lin. T.S., Lan, C.C.E., Tsai, J.C., Wong, T.W., Wei, K.J., Lin, T.X., Chang, N.S. and Sheu, H.M. (2011) Solanum incanum Extract (SR-T100) Induces Cutaneous Squamous Cell Carcinoma Apoptosis through Modulating Tumour Necrosis Factor Receptor Signalling Pathway. Journal of Dermatological Science, 63, 83-92. https://doi.org/10.1016/j.jdermsci.2011.04.003

[33] Yu, S., Sheu, H.M. and Lee, C.H. (2017) Solanum incanum Extract (SR-T100) Induces Melanoma Cell Apoptosis and Inhibits Established Lung Metastasis. Oncotarget, 8, 103509-103517. https://doi.org/10.18632/oncotarget.21508

[34] Wu, J.L., Tang, X.J., Ma, C.J., Shi, Y., Wu, W.Y. and Hann, S.S. (2020) The Regulation and Interaction of Colon Cancer-Associated Transcript-1 and miR7-5p Contribute to the Inhibition of SP1 Expression by Solamargine in Human Nasopharyngeal Carcinoma Cells. Phytotherapy Research, 34, 201-213. https://doi.org/10.1002/ptr.6555

[35] Pee, H.N. (2016) Evaluation of Solamargine as a Therapeutic in Bladder Cancer. Scholar Bank@NUS Repository.

[36] Cham, B.E. (2008) Cancer Intralesion Chemotherapy with Solasodine Rhamnosyl Glycosides. Research Journal of Biological Sciences, 3, 1008-1017.

[37] Sun, L.M., Zhao, Y., Li, X., Yuan, H.Q., Cheng, A. and Lou, H.X. (2010) A Lysosomal-Mitochondrial Death Pathway Is Induced by Solamargine in Human K562 Leukemia Cells. Toxicology in Vitro, 24, 1504-1511. https://doi.org/10.1016/j.tiv.2010.07.013

[38] Xiang, S.T., Zhang, Q.H., Tang, Q., Zheng, F., Wu, J.J., Yang, L.J. and Hann, S.S. (2016) Activation of Ampkalpha Mediates Additive Effects of Solamargine and Metformin on Suppressing MUC 1 Expression in Castration-Resistant Prostate Cancer Cells. Scientific Reports, 6, Article No. 36721. https://doi.org/10.1038/srep36721

[39] Kuo, K.W., Hsu, S.H., Li, Y.P., Lin, W.L., Liu, L.F., Chang, L.C., Lin, C.N., Lin, C.C. and Sheu, H.M. (2000) Anticancer Activity Evaluation of the Solanum Glycoalkaloid Solamargine. Triggering Apoptosis in Human Hepatoma Cells. Biochemical Pharmacology, 60, 865-1873. https://doi.org/10.1016/S0006-2952(00)00506-2

[40] Liang, C.H., Liu, L.F., Shiu, L.Y., Huang, Y.S., Chang, L.C. and Kuo, K.W. (2004) Action of Solamargine on TNFs and Cisplatin-Resistant Human Lung Cancer Cells. Biochemical and Biophysical Research Communications, 322, 751-758. https://doi.org/10.1016/j.bbrc.2004.07.183

[41] Xie, X.D., Zhang, X.M., Chen, J., Tang, X., Wang, M.Q., Zhang, L., Guo, Z. and Shen, W.R. (2019) $\mathrm{Fe}_{3} \mathrm{O}_{4}$-Solamargine Induces Apoptosis and Inhibits Metastasis of Pancreatic Cancer Cells. International Journal of Oncology, 54, 905-915. https://doi.org/10.3892/ijo.2018.4637

[42] Millward, M., Powell, A., Tyson, S., Daly, P., Ferguson, R. and Carter, S. (2005) Phase I Trial of Coramsine (SBP002) in Patient with Advanced Solid Tumors. Journal of Clinical Oncology, 23, 3105.

https://doi.org/10.1200/jco.2005.23.16_suppl.3105

[43] Munari, C.C., De Oliveira, P.F., Campos, J.C.L., Martins, S.P.L., Da Costa, J.C., Bastos, J.K. and Tavares, D.C. (2014) Antiproliferative Activity of Solanum Lycocarpum Alkaloidal Extract and Their Constituents, Solamargine and Solasonine, in Tumor Cell Lines. Journal of Natural Medicines, 68, 236-241.

https://doi.org/10.1007/s11418-013-0757-0

[44] Cham, B.E. (1988) Monograph BEC. Drugs of the Future, 13, 714-716.

https://doi.org/10.1358/dof.1988.013.08.63026 
[45] Punjabi, S., Cook, L.J., Kersey, P., Marks, R. and Cerio, R. (2008) Solasodine Glycoalkaloids: A Novel Topical Therapy for Basal Cell Carcinoma. A Double Blind, Randomized, Placebo-Controlled, Parallel Group, Multicentre Study. International Journal of Dermatology, 47, 78-82. https://doi.org/10.1111/j.1365-4632.2007.03363.x

[46] Cerio, R. and Punjabi, S. (2002) Clinical Appraisal of BEC5. Dermatology Department, Barts and the London NHS, London.

[47] Tambuscio, A., Siliprandi, L., Dario, M., Cham, A., Cham, B. and Bordignon, M. (2016) Treatment of Cutaneous Carcinomas with a Topical Cream Containing Solasodine Rhamnosides: Focus on Efficacy, Compliance and Cosmetic Outcomes. European Academy of Dermatology and Venereology, Congress, Athens, 19-21 May 2016, 248 p.

[48] Cham, B.E. (2007) The Eggplant Cancer Cure: A Treatment for Skin Cancers and New Hope for Other Cancers from Nature's Pharmacy. Smart Publications, USA.

[49] Cham, B.E. (2007) Solasodine Rhamnosyl Glycosides in a Cream Formulation Is Effective for Treating Large and Troublesome Skin Cancers. Research Journal of Biological Sciences, 2, 749-761.

[50] Cham, B.E. (2009) When Does Alternative Become Orthodox? Skin Cancer Treatment with Solasodine Rhamnosyl Glycosides in Its Transitional Stage, a Case Study. Evidence-Based Complementary and Alternative Medicine, 6, 415-420.

[51] Cham, B.E. (2011) Topical Solasodine Rhamnosyl Glycosides Derived from the Eggplant Treats Large Skin Cancers: Two Case Reports. Journal of Clinical Medicine. 2, 473-477. https://doi.org/10.4236/ijcm.2011.24080

[52] Chase T.R. (2011) Curaderm ${ }^{\mathrm{BEC} 5}$ for Skin Cancers, Is It? An Overview. Journal of Cancer Therapy, 2, 728-745. https://doi.org/10.4236/jct.2011.25099

[53] Cham, B.E. (2012) Intralesion and Curaderm ${ }^{\mathrm{BEC} 5}$ Topical Combination Therapies of Solasodine Rhamnosyl Glycosides Derived from the Eggplant or Devil's Apple Result in Rapid Removal of Large Skin Cancers. Methods of Treatment Compared. International Journal of Clinical Medicine, 3, 115-124. https://doi.org/10.4236/ijcm.2012.32024

[54] Cham, B.E. (2013) Topical Curaderm ${ }^{\mathrm{BEC} 5}$ Therapy for Periocular Nonmelanoma Skin Cancer: A Review of Clinical Outcomes. International Journal of Clinical Medicine, 4, 233-238. https://doi.org/10.4236/ijcm.2013.45041

[55] Cham, A. and Cham, B.E. (2015) Treatment of Skin Cancer with a Selective Apoptotic-Inducing Curaderm ${ }^{\mathrm{BEC} 5}$ Topical Cream Containing Solasodine Rhamnosides. International Journal of Clinical Medicine, 6, 326-333. https://doi.org/10.4236/ijcm.2015.65042

[56] Cham, K., Cham, A., Chase, T., Zhou, V. and Cham, B.E. (2015) Treatment of Non Melanoma Skin Cancers: An Intra-Comparison Study of Curaderm ${ }^{\mathrm{BEC} 5}$ and Various Established Modalities. Journal of Cancer Therapy, 6, 1045-1053. https://doi.org/10.4236/jct.2015.612114

[57] Cham, B.E. (2013) Solasodine Glycosides: A Topical Therapy for Actinic Keratosis. A Single-Blind, Randomized, Placebo-Controlled, Parallel Group Study with Curaderm. Journal of Cancer Therapy, 4, 588-596.

[58] Bordingnon, M. (2016) Efficacy and Safety of Curaderm ${ }^{\mathrm{BEC} 5}$ in the Treatment of Basal Cell Carcinoma: A Pilot Study in the Italian Population. European Academy of Dermatology and Venereology Congress, Athens, 19-21 May 2016, 259 p.

[59] Dobrokhotova, F.Z., Betsev, A.F. and Belysheva, T.S. (2016) The Use of Kuraderm Drug in the Treatment of Basal Cell Carcinoma of the Head and Neck. Head and 
Neck Tumors, 6, 22-26. https://doi.org/10.17650/2222-1468-2016-6-3-22-26

[60] Cham, B.E. (2015) Curaderm, Natural Non-Invasive Medication for Skin Cancer. Curaderm Global, Port Vila, Republic of Vanuatu.

[61] Batsev, A.F., Dobrokhotova, V.Z. and Cham, B.E. (2016) Topical Cream Curadermbec5 Treats a Recalcitrant Basal Cell Carcinoma. Clinical Medical Reviews and Case Reports, 3, Article ID: 098. https://doi.org/10.23937/2378-3656/1410098

[62] Cham, B.E. (2020) First in Man Topical Treatment of Melanoma with Solasodine Glycosides in a formulation Curaderm. Journal of Cancer Therapy, 11.

[63] Spochacz, M., Szmczak, M., Chowanski, S. and Adamski, Z. (2020) Solanum Nigrum fruit extract increases toxicity of Fenitrothion-A Synthetic Insecticide, in the Mealworm Beetle Tenebrio molitor Larvae. Toxins, 12, 612-638. 